\title{
Imaging of STAT3 deficiency infectious complications
}

\author{
Alison Haynes $^{a *}$ and David Manson ${ }^{b}$
}

\begin{abstract}
Background: Hyper IgE syndrome (HIES) is a primary immunodeficiency with sporadic, autosomal dominant (STAT3 mutation) and autosomal recessive (DOCK8 and TYK2 mutations) inheritance patterns. HIES is characterized by recurrent Staphylococcus infections including lung infections, skin and visceral abcesses, pulmonary pneumatoceles, mucocutaneous candidiasis, and eczematous rash with associated elevated lgE. Patients with STAT3 mutations have additional features including connective tissue, skeletal, dental, and vascular abnormalities.

Methods: This case series highlights the clinical presentation, radiographic findings, and immunologic investigations of 4 siblings with HIES caused by STAT3 mutation.

Results: Our patients presented with infections including Staphylococcus aureus visceral abscesses, skin lesions, pneumonia with associated pneumatocele, and minimal signs of inflammation such as fever. Additional characteristic features included eczematous rash, scoliosis, fractures, and delayed shedding of primary teeth. Immune investigations were essentially unremarkable apart from elevated IgE and eosinophil counts. Detailed imaging identified infectious processes and associated noninfectious features of STAT3 mutations.

Conclusion: Patients presenting with recurrent Staphylococcal cutaneous and visceral infections, pneumatoceles, candidiasis, and eczematous rash with associated elevated IgE should be investigated for STAT3 mutations. This case series highlights the important role of radiographic imaging to identify infectious processes as well as noninfectious associated features in patients with STAT3 mutations.
\end{abstract}

Statement of novelty: Detailed images of morbidity associated with a STAT3 deficiency.

\section{Introduction}

Hyper IgE syndrome (HIES) is classified as a welldefined syndrome with associated primary immunodeficiency (Al-Herz et al. 2011). The pathogenesis of HIES syndrome is related to defects in Janus activated kinase - signal transducer and activator of transcription (JAK-STAT) signalling pathway (Heimall et al. 2010). HIES can be sporadic or familial with both autosomal dominant and recessive cases described. The autosomal dominant form of HIES is most commonly caused by a mutation in signal transduction and activators of transcription 3 (STAT3) (Holland et al. 2007; Minegishi et al.
2007), whereas the less common autosomal recessive forms are linked to mutations in dedicator of cytokinesis 8 (DOCK8) (Renner et al. 2004; Engelhardt et al. 2009) and tyrosine kinase 2 (TYK2) (Minegishi et al. 2006). STAT3 is involved in the secretion and signalling of a number of cytokines including IL-6, IL-10, IL-11, IL-17, IL-21, and IL-22 (Commins et al. 2008). STAT3 mutations lead to impaired production and signalling of these cytokines, resulting in an abnormal inflammatory response affecting both proinflammatory and anti-inflammatory pathways (Heimall et al. 2010). Furthermore, STAT3 mutations impair the differentiation of naive CD4 $\mathrm{T}$ cells into IL-17 producing Th17
aDivision of Immunology and Allergy, The Hospital for Sick Children, Toronto, Canada; ${ }^{\text {b} T h e ~ D e p a r t m e n t ~ o f ~ D i a g n o s t i c ~ I m a g i n g, ~ T h e ~ H o s p i t a l ~ f o r ~ S i c k ~}$ Children and The University of Toronto, Toronto, Canada

*Corresponding author: Alison Haynes/haynesalison@yahoo.com
Submitted 19 June 2014

Accepted 27 June 2014

Available online 20 August 2014

LymphoSign Journal 1:44-54 (2014)

dx.doi.org/10.14785//psn-2014-0005 
cells, which are important for defence against fungal and extracellular bacteria (Milner et al. 2008). Impairments in IL-17 may contribute to susceptibility to fungal and bacterial infections in HIES patients (Milner et al. 2008). Recent studies have also shown a defect in the number of central memory CD4 and CD8 T cells in HIES patients, resulting in impaired control of Varicellazoster virus and Epstein-Barr virus (Siegel et al. 2011). Some patients have humoral defects with poor specific antibody production to protein and polysaccharide immunizations (Avery et al. 2010) and reduced memory B cells (Speckmann et al. 2008).

HIES secondary to STAT3 mutation is a multisystem disease affecting the immune system, skeletal system, connective tissues, and vasculature. Specifically, STAT3 mutations have been associated with increased susceptibility to infections most commonly secondary to Staphylococcus aureus and candidiasis (Grimbacher et al. 1999a). The most common laboratory findings include an elevation in IgE, with the majority of cases over $2000 \mathrm{IU} / \mathrm{mL}$, and eosinophilia (Grimbacher et al. 1999a). We present 4 siblings with known STAT3 mutations who presented with many classic infectious and noninfectious features described in patients with STAT3 mutations. Such features are outlined with detailed radiographic images and highlight the importance of high equality imaging to aid in the diagnosis and management of patients with HIES.

\section{Results}

\section{Patient 1}

A female patient born at term to nonconsanguineous parents was admitted at the age of 4 months with significant bilateral neck lymphadenopathy. Three weeks prior to admission she had upper respiratory tract symptoms with the absence of fever. Physical examination revealed a $1.5 \mathrm{~cm}$ lymph node in the left upper cervical region, a $2 \mathrm{~cm}$ lymph node in the posterior cervical chain, and two $0.5 \mathrm{~cm}$ lymph nodes behind the left ear. She was also found to have hepatosplenomegaly. An initial ultrasound examination revealed large necrotic bilateral lymph nodes, consistent with lymphadenitis. A liver abscess measuring $5 \mathrm{~cm} \times 6 \mathrm{~cm}$ was noted on the abdominal ultrasound. Excisional lymph node biopsy and aspiration of the liver abscess were positive for Staphylococcal aureus. In terms of additional history, she had severe seborrhea dermatitis, an eczematous rash, and went on to develop retained primary teeth requiring extraction. The patient's father suffered recurrent pulmonary infections, skin abscesses, septic arthritis of the ankle, and an eczematous rash during childhood. Following completion of a course of intravenous antibiotics, she was started on prophylactic sulfamethoxazole-trimethoprim for a suspected primary immunodeficiency.

She remained on prophylactic sulfamethoxazoletrimethoprim until the age of 3 years. She was well until 8 years of age when she presented with abdominal pain and hepatomegaly. Blood cultures were positive for Staphylococcal aureus. Abdominal ultrasound confirmed a liver abscess (Figure 1). She went on to develop recurrent pulmonary infections (Figure 2) and multiple Staphylococcus aureus associated abscesses requiring hospital admissions for intravenous antibiotics and surgical drainage (Figures 2-5). An initial immune evaluation was performed at age 4 months (Tables 1 and 2).

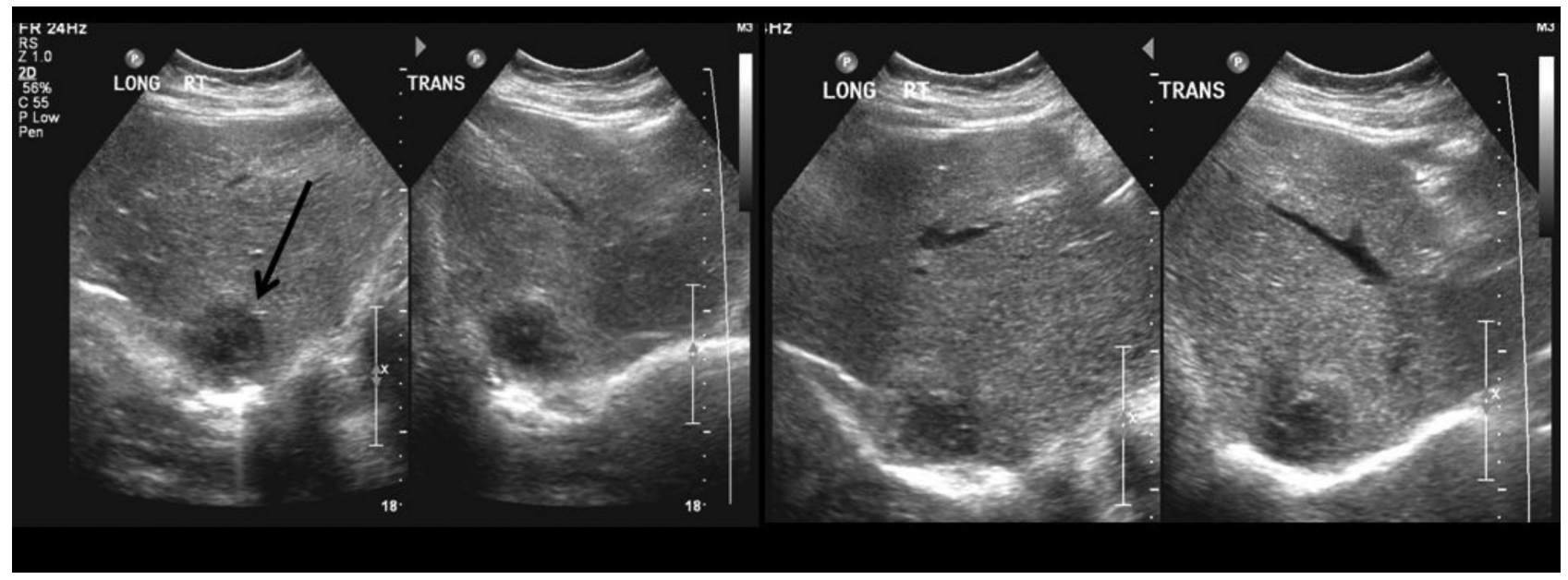

Figure 1: Sonographic images of the complex liver abscess of patient 1. 


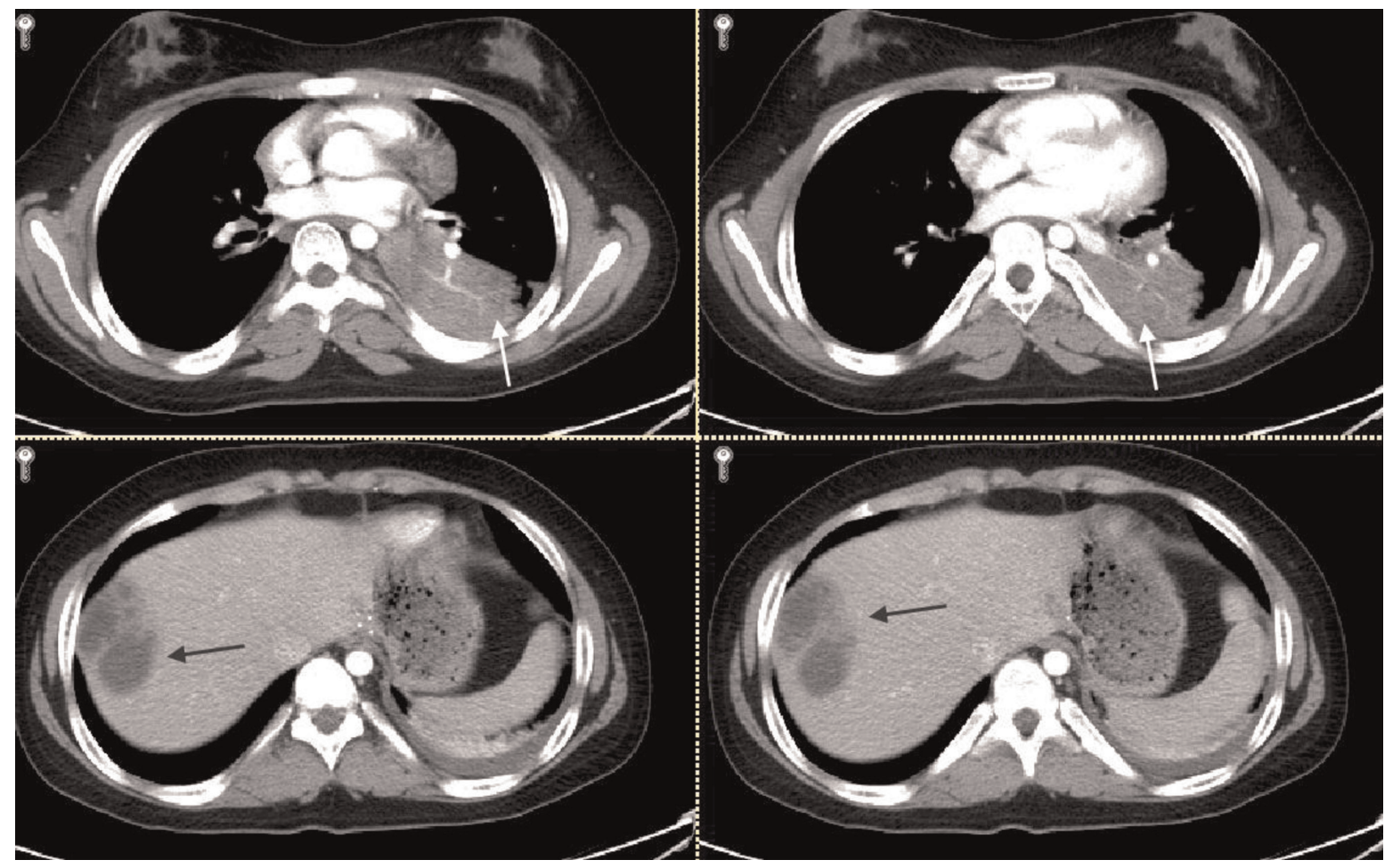

Figure 2: Axial enhanced CT scans demonstrating a left lower lobe consolidation (white arrows) of patient 1. More caudally, there is a focus of decreased enhancement in the liver consistent with an abscess (black arrows).

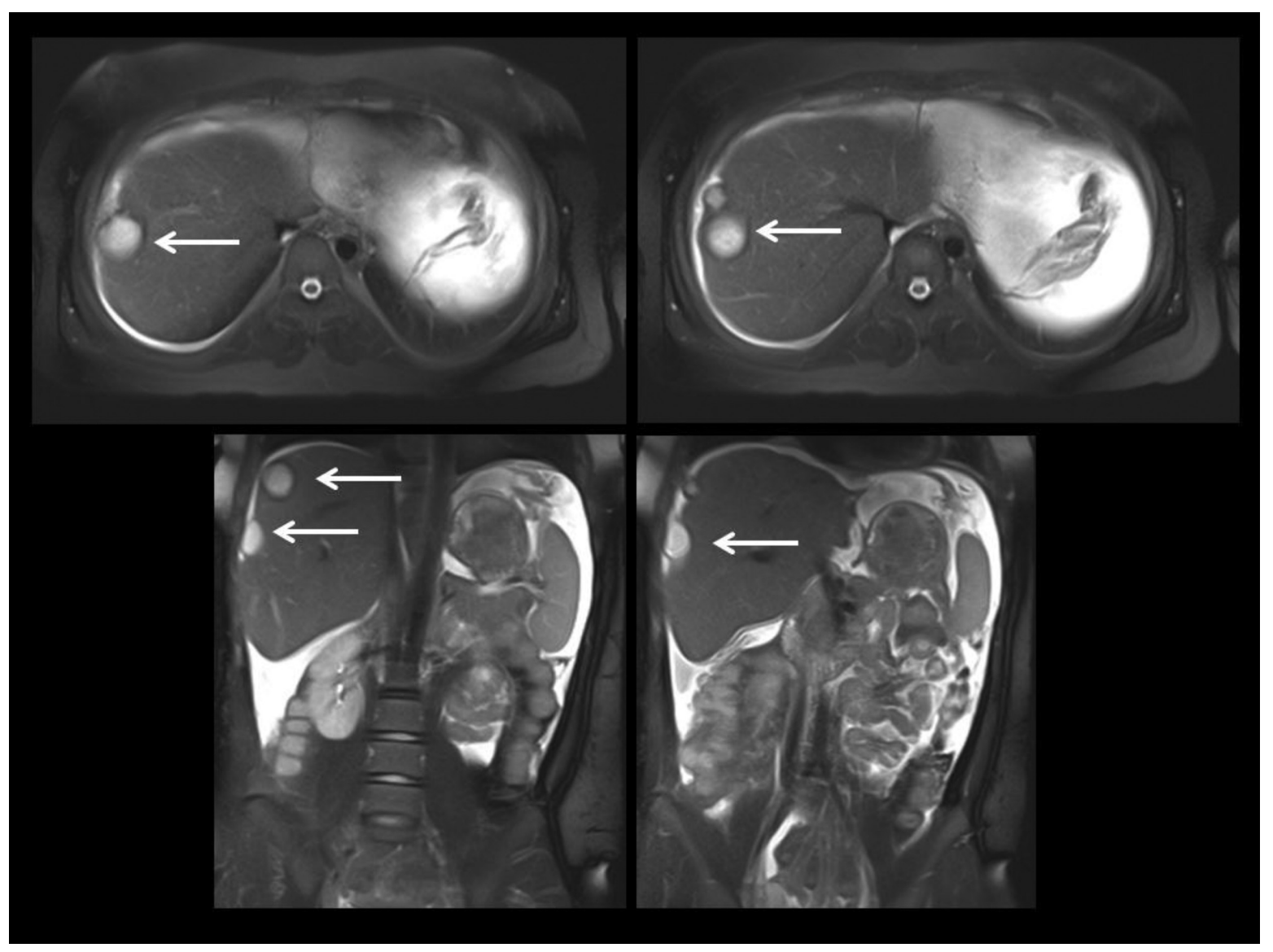

Figure 3: Axial and coronal MRI images demonstrating the liver abscesses of patient 1. 


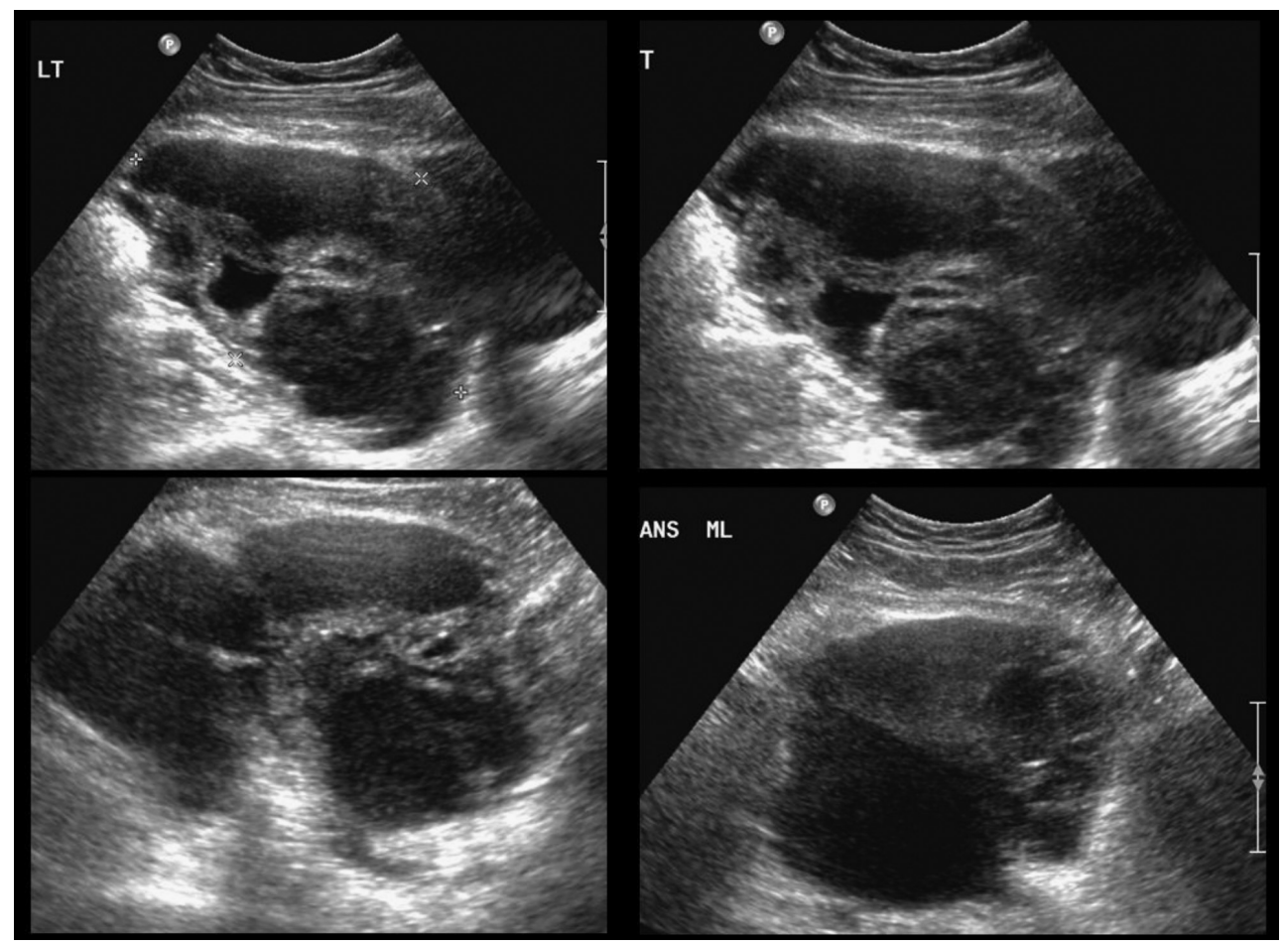

Figure 4: Sonographic images through the pelvis demonstrate multiple complex and septated fluid collections of patient 1.

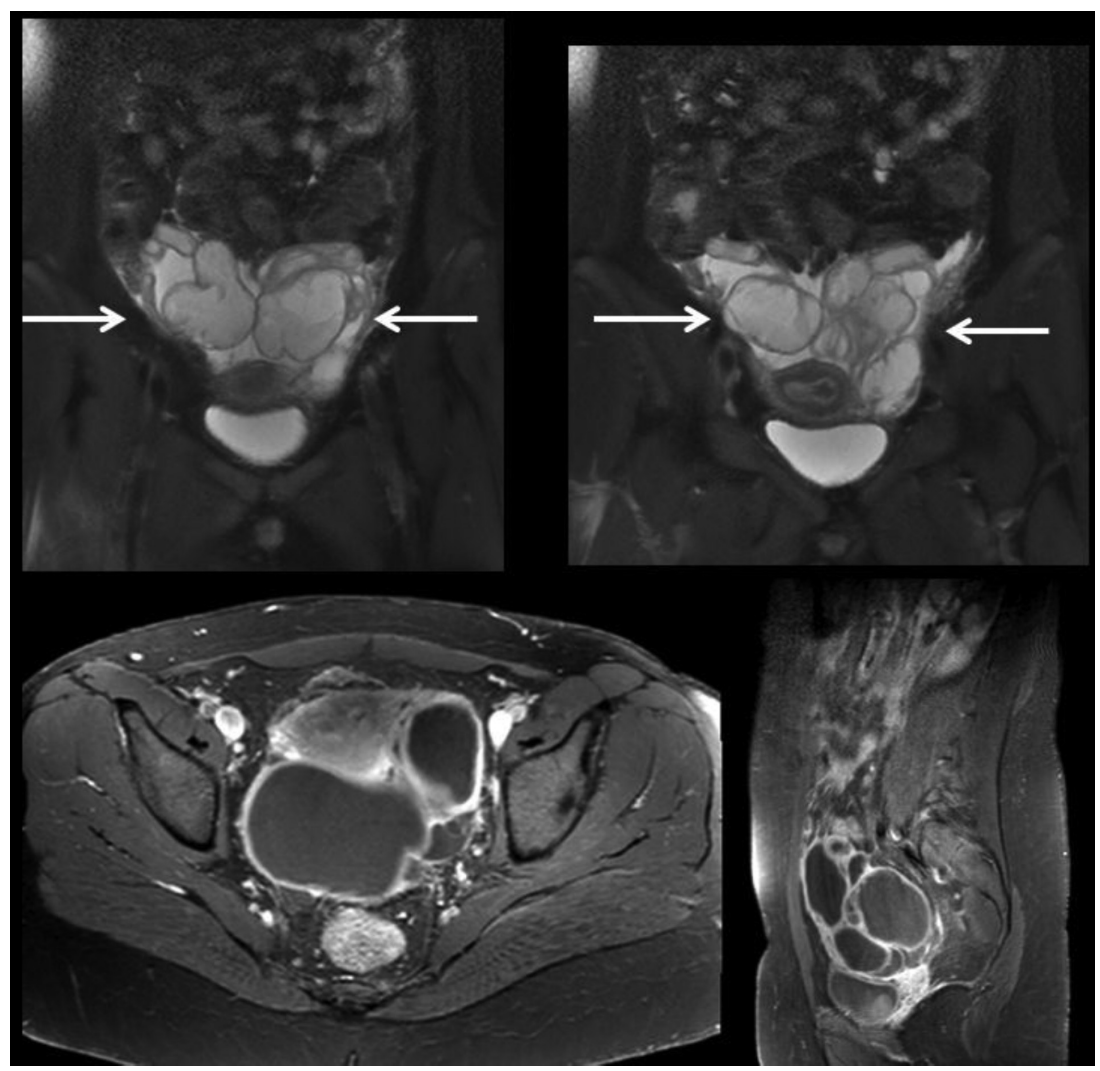

Figure 5: Coronal T2 weighted images demonstrate the complex collections (white arrows) as areas of loculated high signal with surrounding high signal ascites of patient 1. Axial and saggital fat suppressed T1 weighted images after intravenous contrast demonstrates wall enhancement of the collections. 
Table 1: Initial immune evaluation.

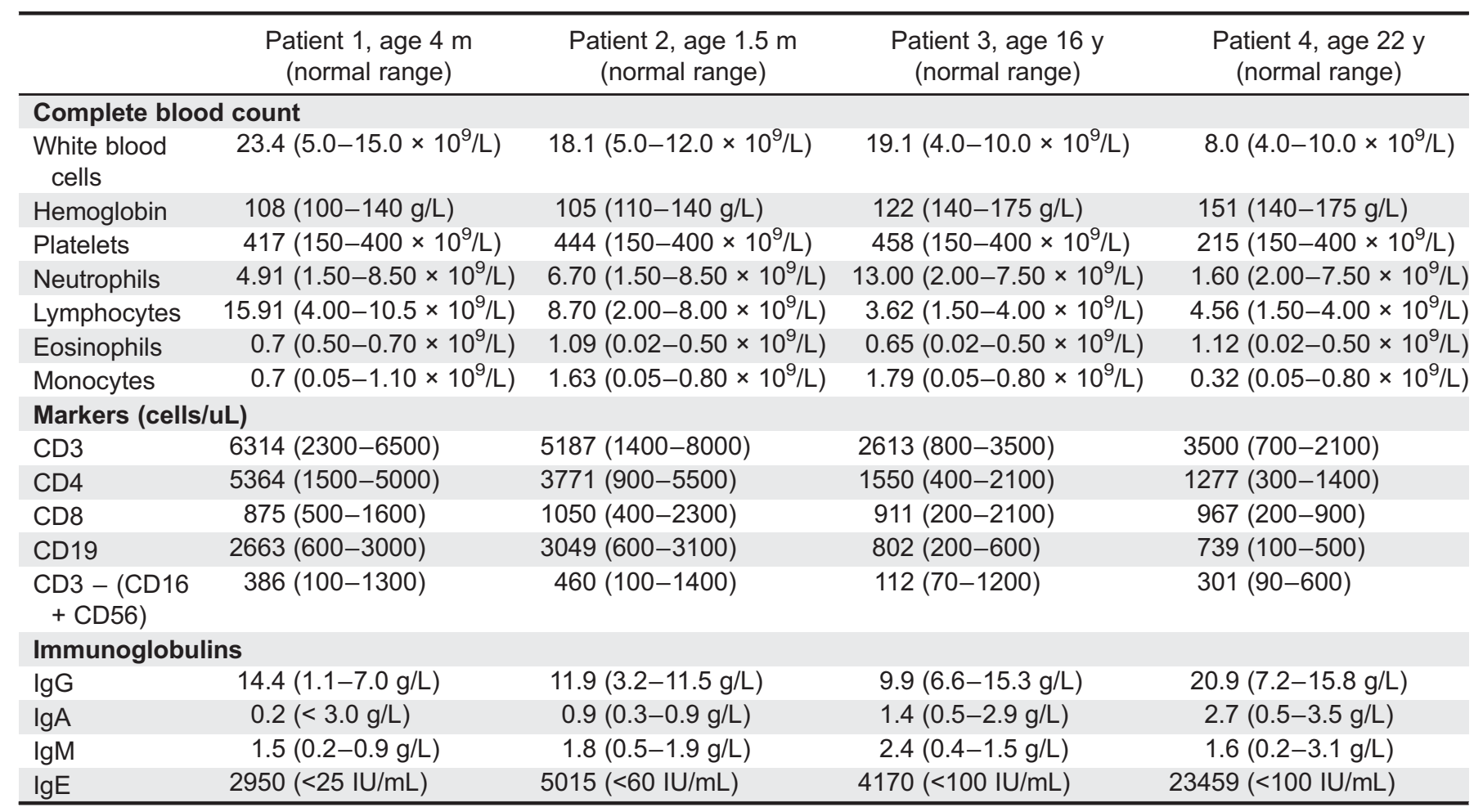

Table 2: Initial immune evaluation.

\begin{tabular}{|c|c|c|c|c|c|}
\hline & Patient 1 , age $4 \mathrm{~m}$ & Patient 2 , age $1.5 \mathrm{~m}$ & Patient 3 , age 16 y & Patient 4 , age $22 \mathrm{y}$ & Normal ranges \\
\hline \multicolumn{6}{|c|}{ Specific antibodies } \\
\hline Tetanus & 2.00 & 2.96 & 0.07 & 0.18 & $>0.1 \mathrm{IU} / \mathrm{mL}$ \\
\hline Measles & ND & Positive & Equivocal & Equivocal & $>1: 16$ \\
\hline Mumps & ND & Positive & Negative & Positive & $>1: 16$ \\
\hline Rubella & ND & Positive & Positive & Positive & $>1: 16$ \\
\hline Varicella & ND & ND & Positive & Positive & $>1: 16$ \\
\hline Polio 1,2,3 & Protective & Protective & ND & ND & $>1: 16$ \\
\hline Isohemagglutinin & ND & Anti A 1:16 & Anti B 1:8 & Anti B 1:8 & $>1: 8$ \\
\hline \multicolumn{6}{|c|}{ Mitogen response* (patient/control) } \\
\hline $\mathrm{PHA}^{\dagger}$ & $141.8 / 62.7$ & $93.7 / 67.5$ & $548 / 616$ & ND & $50 \%$ control \\
\hline
\end{tabular}

*Mitogen response expressed as stimulation index.

$\dagger$ In vitro proliferation of T cells in response to mitogen expressed as stimulation index.

ND, not done.

\section{Patient 2}

The sister of patient 1 presented at 18 months of age with a large soft tissue mass below the angle of the mandible. An ultrasound examination revealed a large cystic nodule measuring $3.7 \mathrm{~cm} \times 2.2 \mathrm{~cm} \times 2.3 \mathrm{~cm}$ (Figure 6). Incision and drainage of the collection confirmed cervical adenitis secondary to Staphylococcus aureus, which was managed with intravenous antibiotics. As an infant she had a facial eczematous rash. At the age of 10 years she developed recurrent skin lesions involving the neck, axillae, and trunk, described as erythematous papules with the expression of a purulent drainage, confirmed to be secondary to Staphylococcus aureus. Additional infections included pneumonia at 1 and 9 years of age. There was also a history of an eczematous rash and retained primary dentation requiring extraction. She developed scoliosis requiring corrective surgery (Figure 7). An initial immune evaluation was performed at 8 months of age (Tables 1 and 2). 

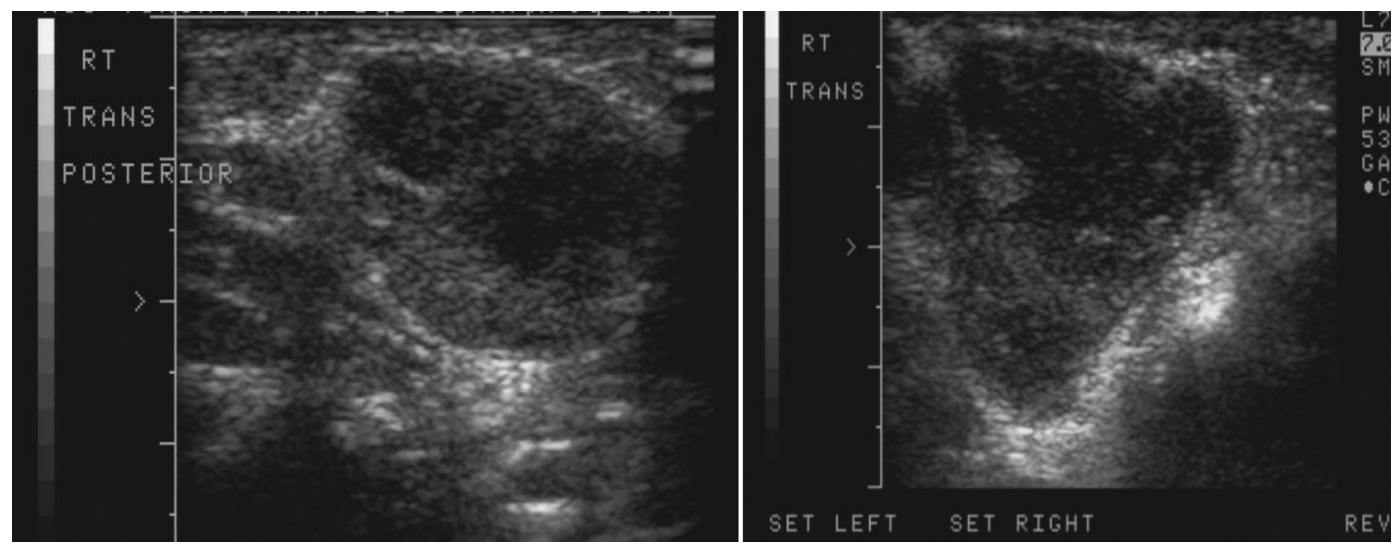

Figure 6: Sonographic images of enlarged cervical lymph nodes that are heterogeneous and demonstrate areas of relative hypoechogenicity, suggesting internal partial liquefaction of patient 2.

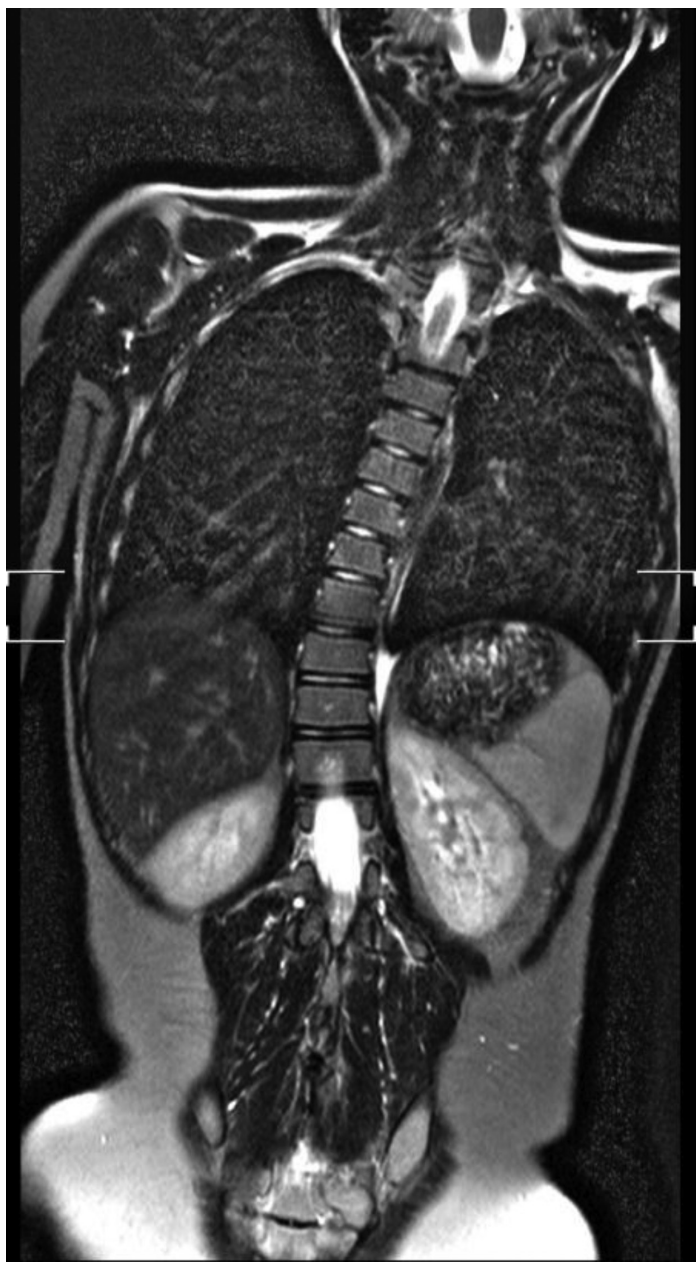

Figure 7: Coronal MRI image demonstrating thoracic scoliosis patient 2 .

\section{Patient 3}

A male child who was the brother of patients 1 and 2, presented at the age of 16 years with a prolonged history

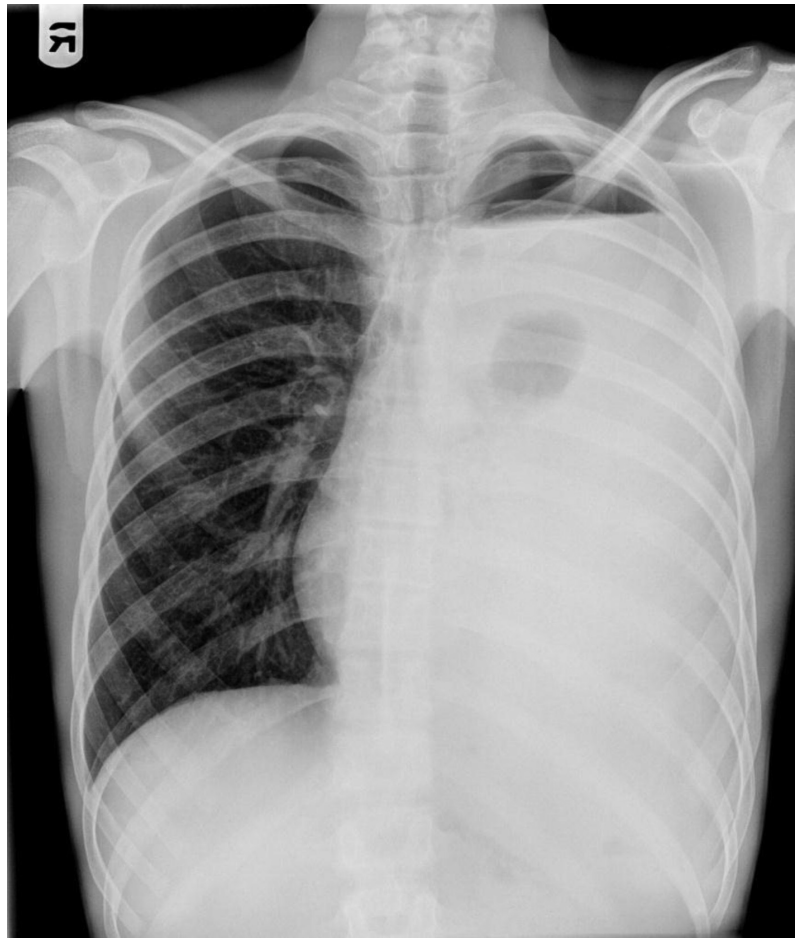

Figure 8: Frontal chest radiograph demonstrates nearcomplete opacification of the left hemithorax. A distinct apical air fluid level suggests a pyo-pneumothorax in patient 3. A small ovoid lucent focus near the left hilum may be loculated air within an abscess.

of cough with absence of fever, which was treated with oral antibiotics that produced a minimal effect. A chest $\mathrm{X}$-ray was completed at presentation (Figure 8) followed by a CT scan of the chest (Figure 9). Chest tube insertion and drainage was positive for Streptococcus anginosus. The previous history was remarkable for a Varicella infection at the age of 4 years with secondary cellulitis. Similar to his siblings, there was also a history of an 

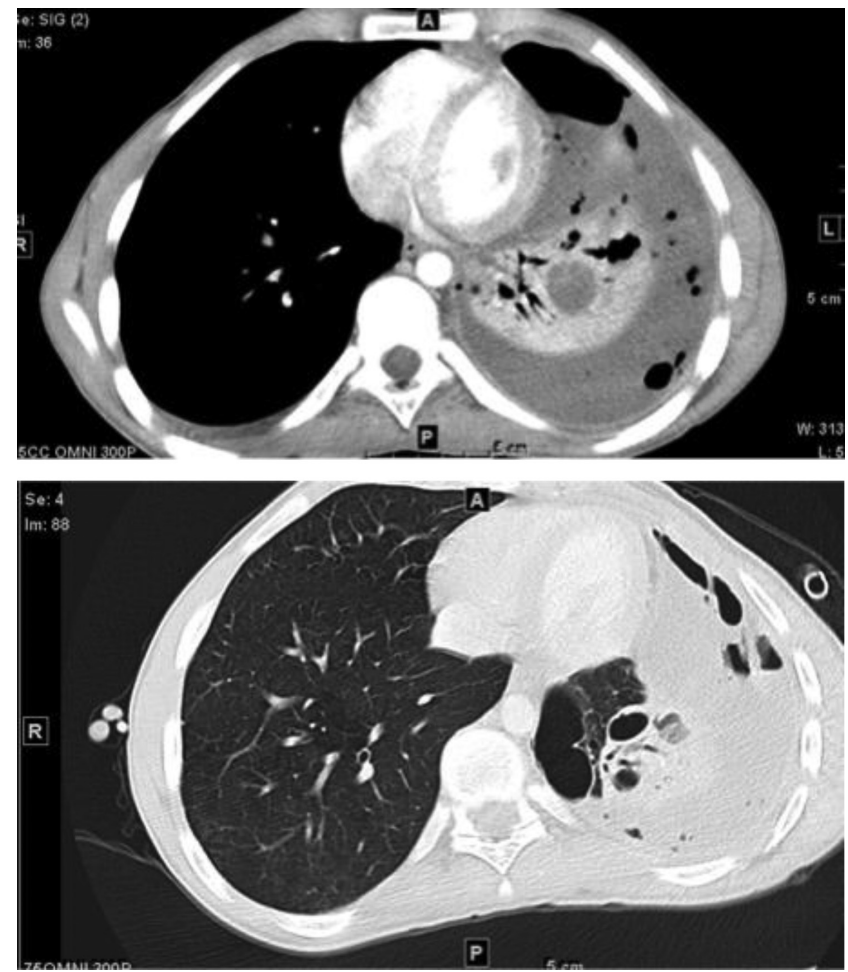

Figure 9: Axial CT images using soft tissue (top) and lung (bottom) algorithms and windows demonstrate enhancing left lung with an internal nonenhancing liquefied focus of a lung abscess patient 3 . Lung windows show a peripheral loculated pneumothorax and internal parenchymal pneumatoceles.

eczematous rash and retained primary teeth requiring extraction. Initial immune evaluation was performed at the age of 16 years (Tables 1 and 2).

\section{Patient 4}

A brother of patients 1, 2, and 3 with a past history of an eczematous rash, retained primary dentation, arm fractures at 1 and 11 years of age following minor trauma, and an episode of pneumonia at 3 years of age. He experienced a significant facial skin infection that required antibiotics and resulted in facial scarring. An intial immune system evaluation at the age of 22 years was remarkable for an increased population of CD3-positive $\mathrm{T}$ lymphocytes that were double-negative for CD4 and CD8 but positive for TCR gamma delta expression. There was also heterogenous expression of CD5, CD7, and CD8 cells. This prompted further investigations for possible T-cell lymphoproliferative process. Physical examination and a CT scan of the chest and abdomen did not show lymphadenopathy. An underlying malignancy was excluded. An initial evaluation of the immune system at the age of 22 years was remarkable (Tables 1 and 2).

\section{Clinical presentation}

This group of 4 siblings presented with infections that are typical for STAT3-associated HIES including abscesses and pneumonia. Staphylococcus aureus associated abscesses occurred in 3 patients and were noted at numerous sites including lymph nodes, liver, lungs, and pelvis, and they required intravenous antibiotics and surgical drainage.

Recurrent skin infections secondary to Staphylococcus aureus occurred in 2 patients.

Patient 1 also had Staphylococcus aureus septicemia. Recurrent pulmonary infections were noted in 3 of the patients, with 1 infection resulting in a significant pulmonary empyema secondary to Streptococcus anginosus and requiring drainage. Additional infections included 1 patient with cutaneous Varicella with secondary cellulitis. A lack of a significant fever was noted with most infections. All 4 patients had an eczematous rash in childhood and retained primary dentation requiring extraction. Additional associated features included scoliosis in patient 2 and fracture with minimal trauma in patient 4 .

\section{Immune evaluation}

A consistent laboratory finding in these patients was an elevated IgE (range 2950-23 $459 \mathrm{IU} / \mathrm{mL}$ ) and high eosinophils (range $0.65-1.12 \times 10^{9} / \mathrm{L}$ ). The remainder of the immune work-up, including lymphocyte immunophenotyping, additional immunoglobulins (IgG, $\operatorname{IgA}, \operatorname{IgM})$, specific antibodies, and mitogen stimulation studies were essentially normal. One patient had a nonprotective tetanus titer that responded to subsequent vaccination (not shown).

\section{Genetic evaluation}

Genetic evaluation confirmed STAT3 mutation in these 4 siblings plus the father (Figures 10 and 11).

\section{Discussion}

The characteristic infectious and noninfectious features of autosomal dominant STAT3 mutation associated HIES are described in these 4 siblings and are demonstrated with supportive imaging. The pathogenesis of these features are felt to be related in part to defects in the secretion and signalling of a number of cytokines involved with the inflammatory response, as 
STAT3

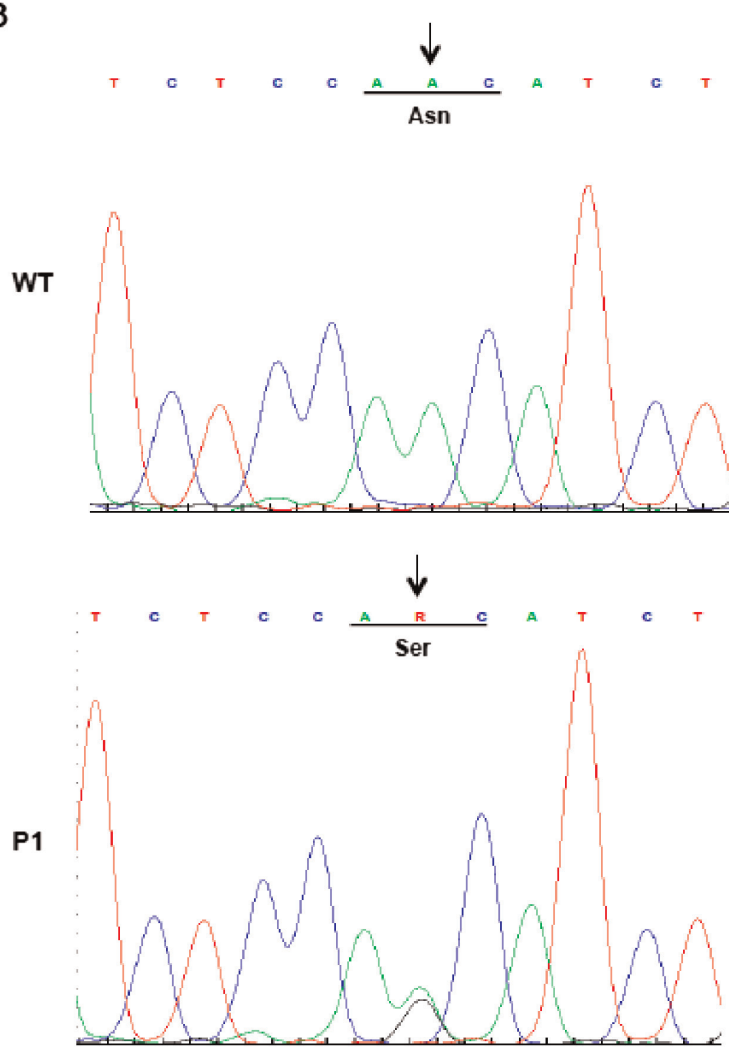

STAT3 NM_139276.2 Mutation c.[1397A>G]; [=] resulting in amino acid change p.[Asn466Ser];[=]

Figure 10: STAT3 NM 139276.2 mutation c.[1397A>G] resulting in amino acid change p.[Asn466Ser].

STAT3

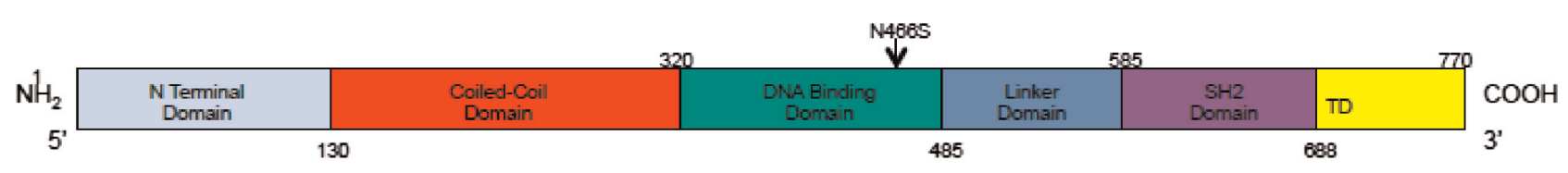

Human STAT3 showing the N Terminal domain, Coiled-coil domain, DNA Binding domain, Linker domain, SH2 domain, Tail Segment domain (TS), and Transactivation domain (TD), their boundaries and the location of the N466S mutation.

Figure 11: STAT3 with major domains labeled with boundary amino acids identified. Disease associated mutation lie within the DNA binding domain.

well as differentiation into effector cells such as Th17 which are important for the defense against bacterial and fungal infections (Milner et al. 2008; Heimall et al. 2010). As demonstrated in our patients, STAT3 mutations lead to susceptibility to sinopulmonary infections, especially due to Staphylococcus aureus, which can progress to bronchiectasis, parenchymal lung damage, and formation of pneumatoceles (Grimbacher et al. 1999a). Colonization of pneumatoceles with Pseudomonas (Grimbacher et al. 1999a), Aspergillus (Vihn et al. 2010) and nontuberculous Mycobacterium (Melia et al. 2009) are also reported. Staphylococcal abscesses of skin, lungs, and other viscera, with minimal signs of inflammation, are classically observed (Grimbacher et al. 1999a) and demonstrated radiographically in our patients. Abscesses are classified as "cold" abscesses with the absence of erythema and warmth, owing to lack of inflammatory response (Hill et al. 1974). Another manifestation of an abnormal inflammatory response observed in these patients is the lack of significant fever or inflammatory markers during infections (Heimall et al. 2010). Patients may present with chronic mucocutaneous candidiasis in addition to bacterial infections (Grimbacher et al. 1999a). Reactivation of Varicella-zoster virus, as shown in patient 3, and infrequently opportunistic infections have been described including Pneumocystis jiroveci (Freeman et al. 2006; Garty et al. 2010), Cryptococcus (Jacobs et al. 1984), and Histoplamosis (Hutto et al. 1988). In addition to infections of the skin, infants present with an eczematous rash with a skin biopsy demonstrating a predominance of eosinophilic infiltration (Olaiwan et al. 2011). 
All 4 of our patients had a description of an eczematous rash early in childhood. Skeletal abnormalities include craniosyntosis (Hoger et al. 1985), fractures with minimal trauma, osteopenia, hyperextensible joints, and scoliosis, as visualized in the imaging for patient 2 (Grimbacher et al. 1999a). Patients have higher rates of vascular abnormalities including hypertension, aneurysms involving coronary and cerebral vessels, and vasculitis (Freeman et al. 2007a, 2011). Additional central nervous system abnormalities described include focal hyperintensities, Chiari 1 malformations, and lacunar infarcts (Freeman et al. 2007a). Defects of the immune system also lead to higher rates of malignancy, in particular non-Hodgkin's lymphoma (Leonard et al. 2004). It was due to this increased risk of malignancy that patient 4 was referred for further evaluation. Patients with HIES have distinct facial features that become more pronounced with age, particularly after puberty. Features include a prominent forehead, deepset wide-spaced eyes, broad nasal bridge with wide fleshy nasal tip, coarse appearing skin, and a high arched palate (Grimbacher et al. 1999a). Delayed shedding of primary teeth leading to double rows of teeth is a unique finding in this patient population (Grimbacher et al. 1999a). All 4 of the patients described had the characteristic facial features that developed over time in addition to the delayed shedding of primary teeth.

A scoring system combining clinical and laboratory findings has been developed to aid in the diagnosis of HIES (Grimbacher et al. 1999b). The most common laboratory findings include an elevation in $\mathrm{IgE}$, with the majority of cases over $2000 \mathrm{IU} / \mathrm{mL}$, and eosinophilia (Grimbacher et al. 1999b). The characteristic elevation in IgE is felt to be an association rather than a contribution to the pathogenesis of HIES. It has been suggested that a defect in the IL-21 receptor signalling results in the elevated IgE levels observed in HIES (Ozaki et al. 2002). Some patients have humoral defects with poor specific antibody production to protein and polysaccharide immunizations (Avery et al. 2010) and reduced memory B cells (Speckmann et al. 2008). Patient 3 had an initial unprotective tetanus titer that responded to repeat vaccination. Additional immune evaluation abnormalities include impaired production of IL-17 (Milner et al. 2008) and decreased central memory CD4 and CD8 cells (Siegel et al. 2011).

Management of STAT3-associated HIES is largely supportive, with aggressive treatment of infections and prevention of infections. Daily prophylactic sulfamethoxazoletrimethoprim is required to prevent staphylococcal infections. As demonstrated in patient 1 , discontinuation of prophylactic antibiotics can lead to significant infections. Antifungal prophylaxis may be used in the case of chronic mucocutaneous candidiasis and pulmonary fungal colonization. Immunomodulating agents such as recombinant human IFN-gamma for severe infections (King et al. 1989) and omalizumab (Chularojanamontri et al. 2009) for skin lesions have been attempted with variable success. A subgroup of patients requires intravenous immunoglobulin replacement owing to poor specific antibody response. Skin care involves the use of topical glucocorticosteroids to inflamed areas and bleach baths to control staphylococcal colonization. Pulmonary infections and resulting complications including pulmonary hemorrhage followed by lymphoma are the leading causes of mortality (Freeman et al. 2007b).

In conclusion, we documented multiple infectious and noninfectious features associated with STAT3 mutations. Patients presenting with recurrent Staphylococcal infections including lung infections, abscesses of skin and organs, pneumatoceles, mucocutaneous candidiasis, and eczematous rash with associated elevated $\operatorname{IgE}$ should be investigated for STAT3 mutation. Detailed radiographic images aid in the diagnosis and management of patients with STAT3-associated HIES.

\section{REFERENCES}

Al-Herz, W., Bousfiha, A., and Casanova, J.L., et al. 2011. Primary immunodeficiency diseases: an update on the classification from the International Union of Immunological Societies Expert Committee for Primary Immunodeficiency. Front. Immunol. 2:54. PMID: 22566844. doi: 10.3389/fimmu.2011. 00054.

Avery, D.T., Deenick, E.K., and Ma, C.S., et al. 2010. B cell intrinsic signaling through IL21 receptor and STAT3 is required for establishing long lived antibody responses in human. J. Exp. Med. 207(1):155-171. PMID: 10053178. doi: 10.1084/jem.20091706.

Chularojanamontri, L., Wimoolchart, S., Tuchinda, P., Kulthanan, K., and Kiewjoy, N. 2009. Role of omalizumab in a patient with hyper-IgE syndrome and review dermatologic manifestations. Asian Pac. J. Allergy. Immunol. 27(4):233-236. PMID: 20232578.

Commins, S., Steinke, J., and Borish, L. 2008. The extended IL-10 superfamily: IL-10, IL-19, IL-20, IL-22, IL-24, IL-26, IL-28, IL-29. J. Allergy. Clin. Immunol. 121(5):1108-1111. PMID: 18405958. doi: 10.1016/j. jaci.2008.02.026. 
Engelhardt, K.R., McGhee, S., and Winkler, S., et al. 2009. Large deletions and point mutations involving the dedicator of cytokinesis 8 (DOCK8) in the autosomal recessive for of hyper-IgE syndrome. J. Allergy. Clin. Immunol. 124(6):1289-1302. PMID: 20004785. doi: 10.1016/j.jaci.2009.10.038.

Freeman, A.F., Avila, E.M., and Shaw, P.A., et al. 2011. Coronary artery abnormalities in hyper IgE syndrome. J. Clin. Immunol. 31(3):338-345. PMID: 21494893. doi: 10.1007/s10875-011-9515-9.

Freeman, A.F., Collura-Burke, C.J., and Patronas, N.J., et al. 2007a. Brain abnormalities in patients with hyperimmunoglobulin E syndrome. Pediatrics. 119(5):e1121e1125. PMID: 17438082. doi: 10.1542/peds.2006-2649.

Freeman, A.F., Davis, J., and Anderson, V.L., et al. 2006. Pneumocystis jiroveci infection in patients with hyper-immunoglobulin E syndrome. Pediatrics. 118(4):e1271-e1275. PMID: 16940164. doi: 10.1542/ peds.2006-0311.

Freeman, A.F., Kleiner, D.E., and Nadiminti, H., et al. 2007b. Causes of death in hyper IgE syndrome. J. Allergy. Clin. Immunol. 119(5):1234-1240. PMID: 17335882. doi: 10.1016/j.jaci.2006.12.666.

Garty, B.Z., Ben-Baruch, A., Rolinsky, A., Woellner, C., Grimbacher, B., and Marcus, N. 2010. Pneumocystis jiroveci pneumonia in a baby with hyper-IgE syndrome. Eur. J. Pediatr. 169(1):35-37. PMID: 19308447. doi: 10.1007/s00431-009-0973-5.

Grimbacher, B., Holland, S.M., and Gallin, J.I., et al. 1999a. Hyper IgE syndrome with recurrent infections - an autosomal dominant multisystem disorder. N. Engl. J. Med. 340(9):692-702. PMID: 10053178. doi: 10.1056/NEJM199903043400904.

Grimbacher, B., Schäffer, A.A., and Holland, S.M., et al. 1999. Genetic linkage of hyper IgE syndrome to chromosome 4. Am. J. Hum. Genet. 65(3):735744. PMID: 10441580 . doi: $10.1086 / 302547$.

Heimall, J., Freeman, A., and Holland, S.M. 2010. Pathogenesis of hyper IgE syndrome. Clin. Rev. Allergy. Immunol. 38(1):32-38. PMID: 19452285. doi: 10.1007/ s12016-009-8134-1.

Hill, H.R., and Quie, P.G. 1974. Raised serum-IgE levels and defective neutrophil chemotaxis in three children with eczema and recurrent bacterial infections. Lancet. 1:183-187.

Hoger, P.H., Boltshauser, E., and Hitzig, W.H. 1985. Craniosynostosis in hyper-IgE syndrome. Eur. J. of Pediatrics. 144(4):414-417. PMID: 4076261.

Holland, S.M., DeLeo, F.R., and Elloumi, H.Z., et al. 2007. STAT3 mutations in the hyper-IgE syndrome. N. Engl. J. Med. 357(16):1608-1619. PMID: 17881745. doi: 10.1056/NEJMoa073687.
Hutto, J.O., Bryan, C.S., Greene, F.L., White, C.J., and Gallin, J.I. 1988. Cryptococcosis of the colon resembling Crohn's disease in a patient with hyperimmunoglobulinemia E - recuurent infection (Job's) syndrome. Gastroenterology. 94(3):808-812. PMID: 3338649.

Jacobs, D.H., Macher, A.M., Handler, R., Bennett, J.E., Collen, M.J., and Gallin, J.I. 1984. Esophageal cryptococcosis in a patient with the hyperimmunoglobulin $\mathrm{E}$ recurrent infection (Job's) syndrome. Gastroenterology. 87(1):201-203. PMID: 6373479.

King, C.L., Gallin, J.I., Malech, H.L., Abramson, S.L., and Nutman, T.B. 1989. Regulation of immunoglobulin production in hyperimmunoglobulin E recurrentinfection syndrome by interferon gamma. Proc. Natl. Acad. Sci. USA. 86(24):10085-10089. PMID: 2513574.

Leonard, G.D., Posadas, E., and Herrmann, P.C., et al. 2004. Non-Hodgkin's lymphoma in Job's syndrome: a case report and literature review. Leuk. Lympoma. 45(12):2521-2525. PMID: 15621772. doi: 10.1080/10428190400004463.

Melia, E., Freeman, A.F., Shea, Y.R., Hsu, A.P., Holland, S.M., and Olivier, K.N. 2009. Pulmonary nontuberculous mycobacterial infections in hyper-IgE syndrome. J. Allergy. Clin. Immunol. 124(3):617-618. PMID: 19733303. doi: 10.1016/j.jaci.2009.07.007.

Milner, J.D., Brenchley, J.M., and Laurence, A., et al. 2008. Impaired $\mathrm{T}(\mathrm{H}) 17$ cell differentiation in subjects with autosomal dominant hyper-IgE syndrome. Nature. 10;452(7188):773-776. PMID: 18337720. doi: 10.1038 /nature 06764 .

Minegishi, Y., Saito, M., Morio, T., and Watanabe, K., et al. 2006. Human tyrosine kinase 2 deficiency reveals its requisite roles in multiple cytokine signals involved in innate and acquired immunity. Immunity. 25(5): 745-755. PMID: 17088085. doi: 10.1016/j.immuni. 2006.09.009.

Minegishi, Y., Saito, M., and Tsuchiya, S., et al. 2007. Dominant negative mutations in the DNA binding domain of STAT3 cause hyper IgE syndrome. Nature. 448:1058-1062. PMID: 17676033. doi: 10.1038/nature06096.

Olaiwan, A., Chandesris, M.O., and Fraitag, S., et al. 2011. Cutaneous findings in sporadic and familial autosomal dominant hyper-IgE syndrome: a retrospective, single-center study of 21 patients diagnosed using molecular analysis. J. Am. Acad. Dermatol. 65(6):1167-1172. PMID: 21703716. doi: 10.1016/j. jaad.2010.09.71.

Ozaki, K., Spolski, R., and Feng, C.G., et al. 2002. A critical role for IL21 in regulating immunoglobulin 
production. Science. 298:1630-1634. PMID: 12446913. doi: 10.1126/science.1077002.

Renner, E.D., Puck, J.M., and Holland, et al. 2004. Autosomal recessive hypergammaglobulin $\mathrm{E}$ syndrome: a distinct disease entity. J. Pediatr. 144(1):93-99. PMID: 14722525. doi: 10.1016/S0022-3476(03)00449-9.

Siegel, A.M., Heimall, J., and Freeman, A.F., et al. 2011. A critical role for STAT3 transcription factor signaling in the development and maintenance of human $\mathrm{T}$ cell memory. Immunity. 35(5):806-818. PMID: 10053178. doi: 10.1016/j.immuni.2011.09.016.
Speckmann, C., Enders, A., and Woellner, C., et al. 2008. Reduced memory B cells in patients with hyper IgE syndrome. Clin. Immunol. 129(3):448-454. PMID: 18835223. doi: 10.1016/j.clim.2008.08.002.

Vihn, C.D., Sugui, J.A., Hsu, A.P., Freeman, A.P., and Holland, S.M. 2010. Invasive fungal disease in autosomal dominant hyper IgE syndrome. J. Allergy. Clin. Immunol. 125(6):1389-1390. PMID: 10053178. doi: 10.1016/j.jaci.2010.01.047. 